\title{
Nursing Experience of 850 Cases on Centralized Cataract Surgery in CDPF Eyesight Recovery Project
}

\author{
Yueqing Miao* \\ Department of ophthalmology, Rudong Traditional Chinese Medical Hospital, Nantong, Jiangsu 226400, China
}

\begin{abstract}
Objective: To explore and summarize the nursing experience of concentrated cataract surgery in CDPF eyesight recovery project. Method: Retrospective analysis of the nursing experience in 850 cases of CDPF cataract patients admitted to the hospital for surgery from June 2008 to July 2014. Results: Through planned, organized education of related knowledge, all patients showed stable emotion and good attitude. They could greatly cooperate in the surgery and master postoperative self-care methods. With fewer complications, their visual acuity was improved to a certain degree. They all reached the standards of blindness exclusion. Conclusion: Organized arrangements, centralized health education, perfect preoperative examination, rational sequencing, careful check and meticulous care were the successful basis of centralized cataract surgery in CDPF eyesight recovery project, which guaranteed a smooth surgery without errors or accidents.
\end{abstract}

\author{
KEYWORDS \\ Cataract surgery \\ CDPF \\ Eyesight recovery
}

\section{Introduction \\ "Millions of Poor Cataract Patients Eyesight Recovery Proj- ect" is jointly led by the Ministry of Health, Ministry of Fi- nance and the China CDPF. It is a major public health ser- vice project listed in the national health reform, designed to help poor cataract patients receive eyesight recovery surgery, solve their problem of blindness due to illness and reduce the burden of their medical treatment [1]. Our hospital has been designated by the County CDPF to carry out Pha- coemulsification combined with intraocular lens implanta- tion since the beginning of 2008. Nearly one thousand cases of cataract blind patients had been cured. This study summa- rizes the nursing experience about 850 cases of CDPF cataract surgery patients from June 2008 to July 2014 as follows.}

\section{Materials and methods}

\subsection{General data}

From June 2008 to July 2014, there were 850 CDPF pa-

Copyright $\odot 2014$ Yueqing Miao

doi: $10.18686 /$ jn.v3i1.2

Received: May 20, 2014; Accepted: July 17, 2014; Published online: September 6, 2014

This is an open-access article distributed under the terms of the Creative Commons Attribution Unported License (http://creativecommons.org/ licenses/by-nc/4.0/), which permits unrestricted use, distribution, and reproduction in any medium, provided the original work is properly cited. ${ }^{\star}$ Corresponding author: Department of ophthalmology, Rudong Traditional Chinese Medical Hospital, Jiangsu Province, Jiangsu Nantong 226400, China. E-mail: yue_qing339@sina.com tients of eyesight recovery project admitted in the ophthalmology ward, including 415 male cases and 435 female cases. Their ages were 55-90 years old. Phacoemulsification and intraocular lens implantation are performed. Their preoperative visual acuity was generally 0.3 or less. All of them were performed monocular surgery. CDPF organized physical examination, centralized admission and centralized operation.

\subsection{Methods}

Before surgery, all patients were checked blood glucose, blood pressure, visual acuity, intraocular pressure, fundus and were performed phacoemulsification and intraocular lens implantation. Intraoperative and postoperative eye condition and visual acuity were recorded in details. The visual acuity of the following 1 week, 1 month and 3-6 months was followed up. Nurse the patients according to cataract care specification strictly, and strengthen care combined with the cataract perioperative standardizing processes made by ophthalmology department. It Included out-patient screening, grasping surgical indications strictly, screening and checking again on admission and before the operation, perioperative care, postoperative medication, observation and prevention of complications, medication guide and follow-up after discharge.

\section{Results}

According to the visual impairment criterion by World 
Health Organization in 1973, the corrected visual acuity of the better eyesight in both eyes $>0.3$ was a normal or nearnormal vision; vision $<0.05$ was blind; $0.05<$ vision $<0.3$ was a low vision. All patients recovered in schedule and reached blindness exclusion standards. 95\% (807 cases) corrected visual acuity reached 0.3 or more, higher than disability standards.

\section{Nursing experience \\ 4.1. Pre-operative nursing}

It mainly contained psychological care and health education. As centralized surgery, patients admitted to hospital often amounted to 30-40 people in one or two days, which increased a lot of pressure to care work. Although nurses canceled rest in whole, it still appeared to be short in full attendance. Most patients were the aged, their mental capacity and ability to understand were poor. They were easy to be nervous and excited, or even induced cardiovascular and cerebrovascular diseases. Therefore, nurses needed to improve the mental capacity of patients and stabilize their emotion. At the beginning of admittance nurses should have an initiative cordial conversation with the patient to promote health knowledge, introduce admission notice, observe and analyze the psychological condition of the patient, and understand the patient's emotional reactions. However, due to a larger number of patients admitted to hospital in one time, it was sometimes difficult to accomplish the mission one by one. Several or even dozens of people were concentrated to make collective health education first, and then nurses separately communicated with individual cases. It not only reduced the workload of nurses, but also improves the efficiency of health education and ensures the quality of health education [2]. Explain to patients about surgical methods, such as surface anesthesia using, short surgery time, small incision, less pain and faster postoperative recovery.

By this way, patients eliminated the psychological pressure, reduced their fear of surgery, increased their trust and rely on nurses. It played an important role on smooth operation and postoperative recovery. "Bandwagon effect" should be properly used in the group event, because following the majority enabled individuals to achieve psychological balance. When individuals realized that they were inconsistent with the majority of people, they tended to be in anxiety and tension, difficult to adapt to the external environment. The behavior of following the majority could alleviate or eliminate such uncomfortable emotion. It helped the individuals be accepted by the majority, which met the need of personal safety and communication. The positive effects of "bandwagon effect" promoted the formation of collective education [3].

Patients and their families fully understood the benefits, effects and possible complications of surgery. It made patients have sufficient psychological preparation and accept psychological support and help from nurses, which facili- tates surgery and postoperative recovery. After admission the nurses should finish the following points:

(1) Nurses should have a comprehensive knowledge of in-patient history and make a good preoperative examination, including: three regular tests, liver and kidney function, blood sugar, whole chest radiograph, electrocardiogram. Grasp their indications of surgery strictly. For the patients with hypertension, diabetes, poor cardiac function, psychological stress, they should be given appropriate treatment to be a stable condition to receive surgery. As concentrated surgery had more patients, nurses should pay special attention to them and mark their special conditions on the surgery list. At the same time, A-ultrasound and Bultrasound check should be made to understand the situation of fundus oculi and axis oculi, calculate IOLs degree, in order to select the appropriate crystals.

(2) Preoperative visit was made 1 day before surgery to determine the operation sequence, distribute operational plates and check whether the patient's name, bed number, plate was consistent with operation order sheet. At the same time make a good explanation to the patient warmly and kindly. Elderly patients were generally suffering from hearing loss, poor comprehension and slow response. The preoperative visit needed meticulous and targeted psychological counseling to improve the sense of trust to doctors and nurses and reduce their psychological stress.

(3) Before surgery, patients should maintain a normal daily life, avoid cold, keep the stable state of mind to avoid fluctuations in blood pressure and blood glucose because of excitement [4].

(4) 3 days before surgery, drop antibiotic eyedrops to control local focus of eye infection. For patients with clear eye infection, it is inappropriate to surgery.

(5) Keep urine and stool unobstructed before surgery. For patients suffering from constipation, stiparolytic medicine or enema surgery can be used repeatedly to prevent eye congestion caused by postoperative constipation.

(6) Train patients to gaze fixed light repeatedly before surgery, and make the correct eye movements according to the requirements in order to cooperate with smooth operation.

\subsection{Intraoperative Care}

(1) Call the number according to preoperative surgical plates, check whether the patient's name, bed number, plate was consistent with operation order sheet and get ready to enter the operating room. (2) Check the patient's name and the affected eye, mark on skin with methylene blue above the affected eye. After the correct checking by nurse and patients, arrange patients to enter the surgical waiting area. (3) Drop tropicamide compound into the affected eye. After full mydriasis, drop oxybuprocaine eye drops as polydocanol. Rinse the conjunctival sac with $0.25 \%$ povidone iodine diluted. At the same time inform the surgical procedures and intraoperative precautions, comfort the 
nervous patient, and arrange the patient to enter the operation room. (4) Assist the patient supine on the operating table, check the patient's bed number, name, affected eye, IOLs degree again. (5) Closely observe the in-patient blood pressure, heart rate, pulse, respiration, etc. In case of change, take emergency measures to ensure the safety of operation. (6) Monocular dressing after surgery, and escort patients to the ward by someone.

\subsection{Postoperative Care}

(1) General care: Patients were in supine or semi-recumbent position. Nurses observed the changes in vital signs of patients. Strengthen the observation of the local conditions of the affected eye. In case of persistent eye pain or bleeding, report to the doctors immediately. Following the doctor orders, nurses gave oral antibiotics to prevent postoperative infection on that day after surgery. On the second day after surgery, eye drops were used until the inflammation subsided. Keep the eye clean. Gently pull the lower eyelid when drip eyedrops and check local conditions of the affected eye at the same time [5].

(2) Psychological care: comfort and encourage the patient cordially, inform the postoperative precautions and methods beneficial to the surgery eye recovery, and give a written Notice to patients, in order to relief their ideological burden to cooperate with treatment actively.

(3) Life care: the patient was told to have appropriate bed rest and to avoid excessive head movement. Keep appropriate warm to avoid cold, coughing or sneezing. Stop laughing loudly. Carefully avoid hitting surgery eye as activities. Diet should be light and digestible, avoid alcohol and spicy food, have more fresh fruit and vegetables, and keep the stool smooth.

(4) Discharge guidance: patient was asked to notice eye health and use clean towels to wash face. Do not rub the eye and drip the eye drops in time. The correct eye drops way was taught to patients. Avoid heavy physical exertion bend within three months. Keep the stool smooth. Reexamine regularly as doctors ordered. In case of eye pain, tearing, redness, visual acuity decreased, foreign body sensation, patients should seek for medical advice immediately. Within two weeks after discharge, understand patients' recovery by telephone follow-ups.

\section{Discussion}

Cataract is the first cause of blindness among the aged in China [6]. With the faster pace of China's population aging, the incidence rate of cataract increases every year. At present, there is no drug can effectively prevent and slow down the development of lens opacities of cataract. Surgery is still the best way for cataract patients to recover eyesight [7]. It is urgent to treat cataract of the aged. The centralized screening and treatment of cataracts gradually increase, which ask for improving the requirements for care.
In cataract centralized eyesight recovery project, its typical features are the quantity of surgery patients is more, but their length of stay in hospital is shorter. Therefore, the traditional care model is not enough to meet the need of such a centralized admission-surgery-discharge flow, and it asks for more strict requirements to care. We take the model of collective health education, combined with comprehensive, standardized care during perioperative period, so it is organized, busy but not chaotic during the period of cataract centralized eyesight recovery surgery, which ensures the safety and quality of care work. (1) Ward nurses are limited, so nursing work is in high intensity. It is the key for achieving high-quality care level to strengthen the training of eye care basic theory and skills and to improve the overall quality of the nursing staff. Not only pay attention to every aspect of quality, such as the admission care, assistance check, surgical transport, preoperative and postoperative care, but also protect the care safety of each patient. (2) It is the key for improving the efficiency and safety of operation to establish a convenient and reasonable medical care process and have someone responsible for every aspect. Our department strengthens care management and arranges all-level nurses reasonably with a clear division of labor. With solidarity and cooperation, someone is responsible for each stage from patient admission, preoperative and postoperative care and discharge guidance, so that the care quality of all aspects have been protected. (3) Avoid the occurrence of infections, especially crossinfection. Strict aseptic technique practice is another key point in care management [8]. (4) The establishment of follow-up files, regular follow-up telephones and urging patients re-check, improve patients' compliance with re-check, which ensure the middle-term and long-term complications of patients receive treatment in time and develop good habits of eye protection by patients themselves.

In recent years, we have created a team combined with medical treatment and nursing as a whole since cataract project of CDPF eyesight recovery project was carried out. A good, cooperative, high quality and rich in medical and nursing experience team serves as the basis to ensure the success of centralized cataract-eyesight-recovery surgery with high quality in primary hospital.

\section{References}

1. Zhang SS, Jia S, Cao ZJ. Cross-sectional study on blindness and low vision among the elderly in xiangcheng district, suzhou city. Journal of Occupational Health and Damage. 2014;29(2):75-77.

2. Xiao HM, Zhi CM, Ye WP. Implementation and evaluation of group health education mode in cataract department. Chinese Journal of Practical Nursing. 2010;26(9):314-315.

3. Zhu LY, Wang S. Prevent and control the harm of bandwagon effect on group decision. Adult Higher Education Journal. 2004;9(1):48-50. 
4. Lin $\mathrm{Y}$, Pei $\mathrm{H}$, Zhang HY. The bandwagon effect in the application of recruiting blood donors. Journal of Community Medicine. 2006;24:30-31.

5. Yang L, Lin L, Qiu Y. Care of cataract ultrasonic emulsification and artificial lens implantation. Chinese Medical Journal Today, 2003;3(12):81.

6. Kui TX, Qiu BG, Lv WS. Modern gerontology. Zhengzhou
University Press. 2001:458-459.

7. Chen $X$. Surgical nursing for the treatment of 88 cases of age-related cataract. Chengdu Medicine. 2002;28(4):235-236.

8. Editorial Department of Ophthalmology in China. Resolutely put an end to cataract surgery iatrogenic infection of mass events. Ophthalmology in China. 2006;15(1):7-8. 